\title{
MATERIAL DAMAGE TEST WITH 450 GeV LHC-TYPE BEAM
}

\author{
V. Kain, K. Vorderwinkler, J. Ramillon, R. Schmidt, J. Wenninger, CERN, Geneva, Switzerland
}

\begin{abstract}
The design of LHC protection elements is based on assumptions on damage levels, which are derived from simulations. A dedicated experiment was carried out to crosscheck the validity of this approach by trying to damage material in a controlled way with beam. The impact of a $450 \mathrm{GeV}$ beam extracted from the SPS on a specially designed high- $Z$ target with a simple geometry, comprising several typical materials used for LHC equipment, was simulated. The beam intensities for the test were chosen to exceed the damage limits of parts of the target. Results of the simulations are presented and compared with test results.
\end{abstract}

\section{INTRODUCTION}

An important prerequisite for the design of the LHC machine protection system is the damage threshold when losing beam in equipment. In general, damage levels are derived from simulations - especially for LHC energies and intensities. The most common approach is to use static energy deposition calculations [1], e.g. with FLUKA [2]. Dynamic effects such as bunch structure and shock waves are not taken into account.

This paper describes an experiment that was carried out to compare predictions for the damage level with the outcome of a beam test. The SPS beam with parameters as for LHC injection was extracted at $450 \mathrm{GeV}$ into the the beam line TT40, which is the upper part of one of the transfer lines between the SPS and the LHC. The beam impacted on a specially designed target. This paper presents the results of the test and a comparison with data from simulations.

\section{CONTROLLED DAMAGE TEST}

A hadron beam impacting on material interacts with the target particles and generates a particle shower, depositing energy in the material. Energy deposition leads to temperature rise and hence possibly to damage. The transverse extent of the shower increases with the penetration depth. The energy deposition is maximum at a certain distance in the target; in solid copper the maximum is reached after about $15 \mathrm{~cm}$ for a $450 \mathrm{GeV}$ proton beam. Damage in the target is a function of the longitudinal coordinate (parallel to the beam axis) as well as transverse coordinates (perpendicular to the beam axis).

The target consisted of a series of tightly packed metal plates, see Fig. 1. This structure allows to understand the longitudinal development of damage by investigating the different plates.
Metals such as copper and stainless steel $(316 \mathrm{~L}, \mathrm{IN}-$ CONEL) commonly used in the LHC were chosen for the plates. In order to have a wide range of melting points, zinc with its comparatively low melting point was also included.

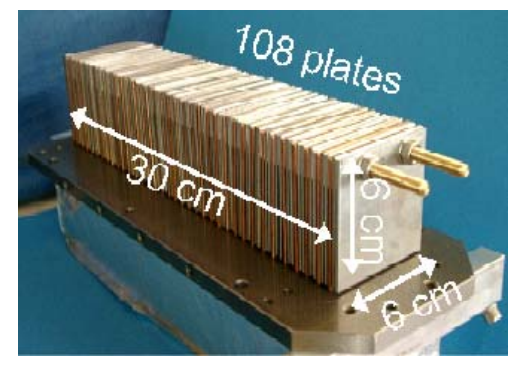

Figure 1: Core of the target for the damage test in TT40.

The dimensions of the plates were $6 \mathrm{~cm} \times 6 \mathrm{~cm} \times 2 \mathrm{~mm}$. Placeholders of $0.5 \mathrm{~mm}$ thickness were installed between two plates to avoid plates sticking together in case of melting. In total the target consisted of 108 plates resulting in an overall length of about $30 \mathrm{~cm}$.

A special arrangement of the materials in the target was chosen. Packages of three plates with different materials, always in the same sequence, were assembled and installed one behind the other. Each package consisted of Zn, stainless steel and $\mathrm{Cu}$, where $\mathrm{Zn}$ was always the first and $\mathrm{Cu}$ always the last plate (every third stainless steel plate was an INCONEL plate). In this way every material experienced every stage of the particle shower.

Numbers which were engraved on the plates defined the plate's longitudinal position for later analysis.

To limit irradiation of the environment, a double confinement was built around the target. The core with the plates was wrapped in a Ti-foil to protect the outer Alconfinement from possible molten metal droplets. The airtight outer container was equipped with a Ti entrance and exit window.

The target could be moved in and out of the beam and to different impact locations by a stepping motor. $\mathrm{An}^{\mathrm{Al}_{2} \mathrm{O}_{3}}$ screen installed on the outer confinement in front of the entrance window was used to steer the beam on the target. Fig. 2 shows the target with confinement, screen and motor installed in front of the beam stopper in TT40 (TED-TT40).

\section{FLUKA SIMULATIONS}

FLUKA simulations for energy deposition were used to define 4 different proton intensities, A, B, C and D, which should cause melting on parts of the plates. Intensity A was chosen to be below the damage limit of all the materials, intensity B should only cause melting of Zn plates, intensity 


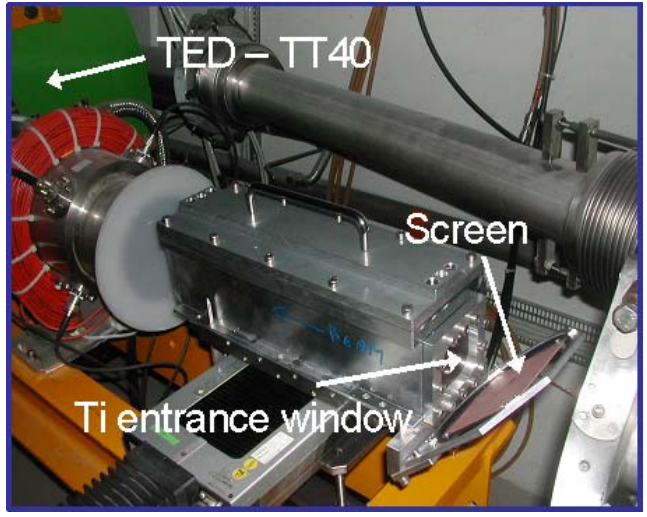

Figure 2: The target installed in TT40 in front of the TEDTT40 in air.

$\mathrm{C}$ of $\mathrm{Zn}$ and $\mathrm{Cu}$ plates and intensity $\mathrm{D}$ should melt $\mathrm{Zn}, \mathrm{Cu}$ and $316 \mathrm{~L}$. INCONEL should stay intact for all chosen intensities.

\section{Simulation Results}

The geometry of the target was implemented in FLUKA including every relevant detail of the double confinement, see Fig. 3. The simulations were done for the nominal R.M.S beam size at the location of the target in TT40: $\sigma_{x}=1.1 \mathrm{~mm}$ and $\sigma_{y}=0.6 \mathrm{~mm}$. Fig. 4 shows the energy

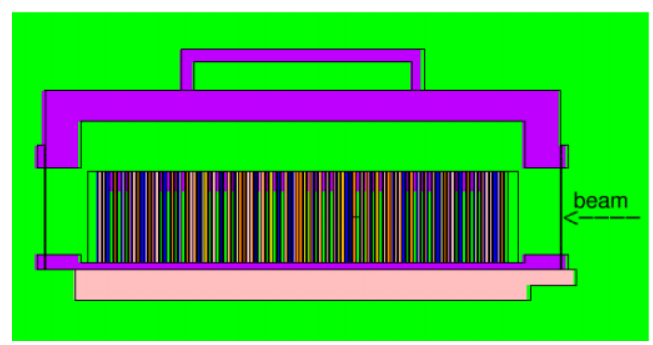

Figure 3: The model of the target implemented in FLUKA.

deposition for the different plates in the shower maximum. The errors are smaller than $8 \%$.

The temperature rise is obtained from the energy deposition using the temperature dependent heat capacity for each material. The result for $\mathrm{Cu}$ is given in Fig. 5 .

Table 1 gives for each intensity and material the first plate number where melting is expected. The heat of fusion, the extra amount of energy leading to the phase transition at the melting point, is taken into account.

\section{RESULTS OF EXPERIMENT - COMPARISON WITH SIMULATION}

Beam with four different intensities A, B, C and D was extracted on the target in four shots on four horizontal target locations on November 8, 2004.

The first inspection of the target after the test and after having removed the confinement showed no damage in the

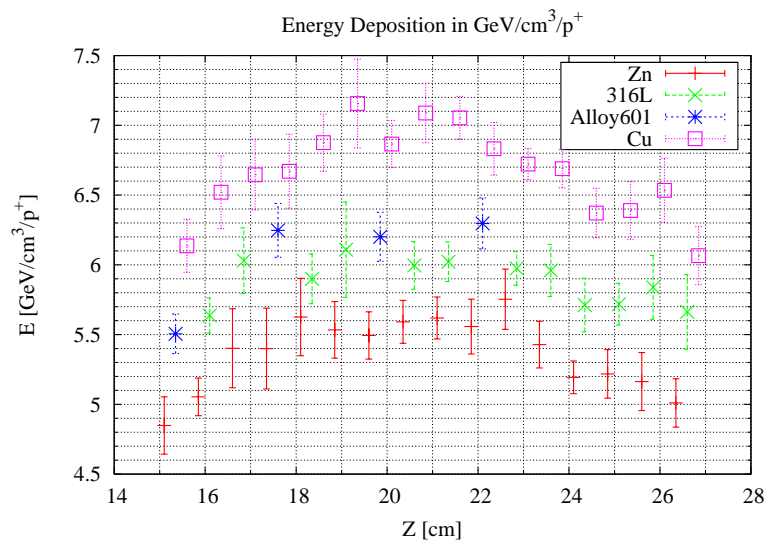

Figure 4: Energy deposition in different plates in the maximum of the shower.

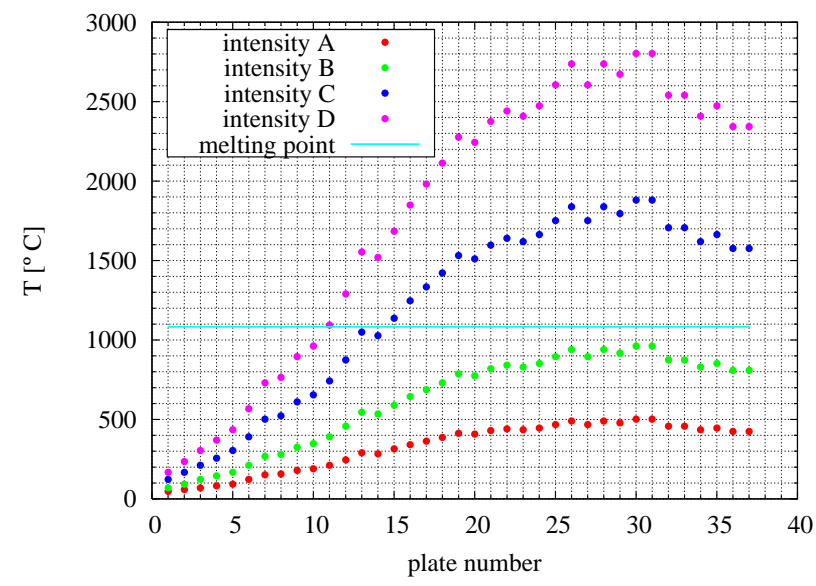

Figure 5: FLUKA results for the temperature in the $\mathrm{Cu}$ plates for the different intensities.

entrance part and more and more damage towards the exit face, as expected. Visual inspection of the target plates was used to check whether there was any damage. No stress related damage had occurred, no cracks or twisting was noticed.

The emittance during the test was about $15 \%$ smaller than assumed for the simulations. With the uncertainties of the beta function at the target location and intensity, an agreement between simulation and experiment better than 20 to $30 \%$ cannot be expected.

Every plate was photographed for further analysis. The

Table 1: FLUKA results

\begin{tabular}{|l|c|c|c|c|c|}
\hline Intensity & protons & \multicolumn{5}{|c|}{ melting starting in plate NR. } \\
\cline { 3 - 6 } & {$\left[10^{12}\right]$} & $\mathrm{Zn}$ & $\mathrm{Cu}$ & $316 \mathrm{~L}$ & INCONEL \\
\hline $\mathrm{A}$ & 1.3 & - & - & - & - \\
\hline $\mathrm{B}$ & 2.6 & 16 & - & - & - \\
\hline $\mathrm{C}$ & 5.3 & 9 & 18 & - & - \\
\hline $\mathrm{D}$ & 7.9 & 7 & 12 & 23 & - \\
\hline
\end{tabular}



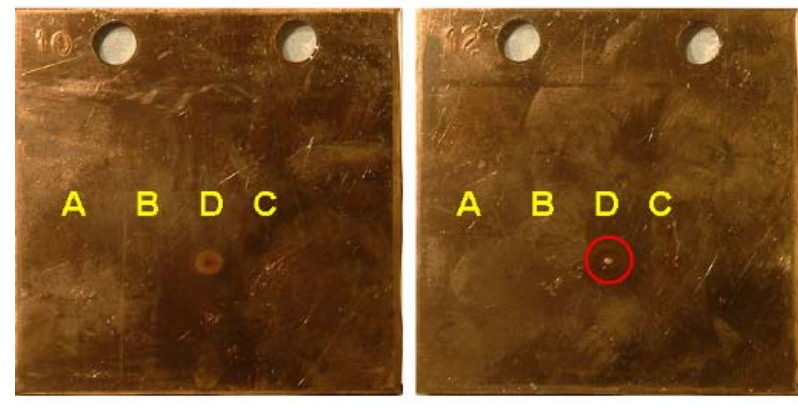

Figure 6: Cu plates 10 and 12. The observation agrees with the simulation.

longitudinal development of damage in the target was compared with the simulation predictions. Fig. 6 and 7 show $\mathrm{Cu}$ plates after the irradiation, the yellow letters indicate the chosen intensity for the impact location. Plate 10 in Fig. 6 only shows discoloration due to heating for intensity $\mathrm{D}$ but no damage, whereas plate 12 just started to melt for the same shot. These results agree well with the simulation results. Other examples are plate 16 and 17 in Fig. 7. Plate 16 only melted for intensity D, plate 17 also shows melting for intensity $\mathrm{C}$. The simulations, however, had predicted that plate 18 and not plate 17 should be the first $\mathrm{Cu}$ plate melting with intensity $\mathrm{C}$. The discrepancy between simulation and experiment in the longitudinal position of the damaged plates for this case is less than $5 \mathrm{~mm}$.

For $\mathrm{Zn}, \mathrm{Cu}$ and INCONEL, the outcome agrees reasonably well with the data from the simulations. For $\mathrm{Cu}$ and $\mathrm{Zn}$, discrepancies in the order of $5 \mathrm{~mm}$ for the location of the damage were observed. INCONEL did not melt, as predicted. According to the simulation, 316L should have melted from plate 23 onwards, but stayed intact for all intensities. The reason for this is very likely the uncertainty on the heat capacity of this alloy.
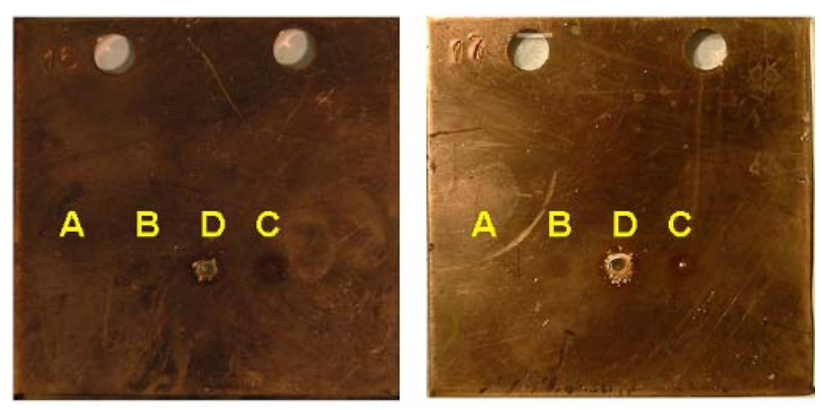

Figure 7: $\mathrm{Cu}$ plates 16 and 17 after the irradiation.

Fig. 8 shows the radii of the damaged area for $\mathrm{Zn}$ plates. The experimental data is in blue. The red points show the radius of a circle where the melting point is exceeded according to the simulation, and the green points correspond to the radius of a circle where the energy deposition exceeds both melting point + heat of fusion. The measured curve seems to be systematically closer to the pre- dicted radii for the regions where the melting point of $\mathrm{Zn}$ is reached. A possible explanation is that the solid material at the melting point becomes soft enough to be moved outwards by the shock wave generated by the beam impact. This effect cannot be described with static energy deposition simulations only, other tools would have to be used [3].

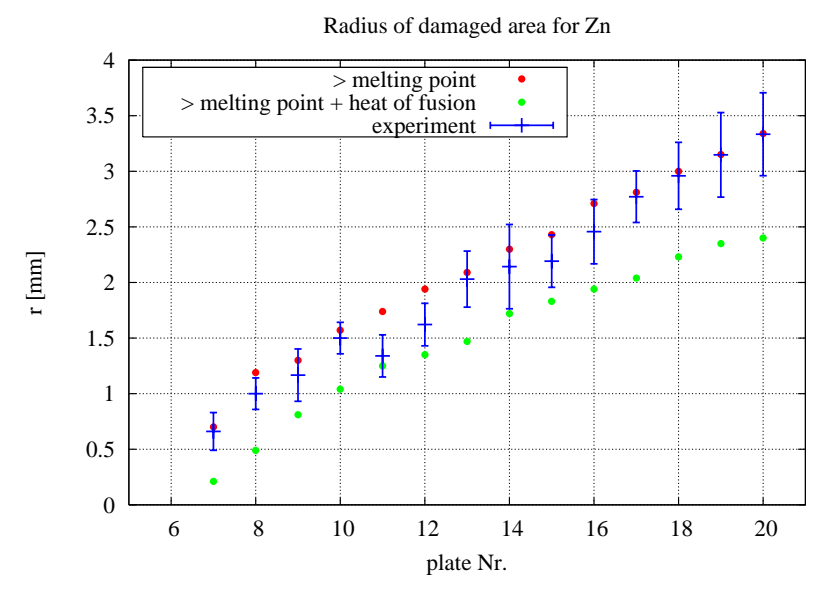

Figure 8: Comparison of radius of damaged area with simulation for $\mathrm{Zn}$.

\section{CONCLUSIONS}

The results of the controlled damage test show reasonable agreement with the simulations. $\mathrm{Zn}-, \mathrm{Cu}-$ and INCONEL-plates are damaged at the predicted locations within the error bars. The transverse extent of the damaged area on the $\mathrm{Zn}$ - and $\mathrm{Cu}$-plates could be predicted within $30 \%$. The outcome of the experiment gives confidence that beam induced damage limits for simple geometries can be adequately predicted with simulations. It is worth mentioning that no stress induced damage was observed when the energy deposition led to a significant temperature increase but remaining below the melting point.

\section{ACKNOWLEDGEMENTS}

This paper is based on the work of many colleagues helping with preparing and carrying out the experiment. We want to express our gratitude.

\section{REFERENCES}

[1] J.M.Zazula, G.R.Stevenson, E.Weisse, Energy Deposition by SPS Beam in Targets of Various Materials, CERN-SL-93-47BT, 1993.

[2] A.Fasso et al., The Physics Models of FLUKA: status and recent development, CHEP 2003, LA Jolla, California, 2003.

[3] N.A.Tahir et al., "The CERN Large Hadron Collider as a Tool to study High-Energy Density Matter", Physical Review Letters, April 2005. 